\title{
Redes sociais de dois modos: aspectos conceituais ${ }^{1}$
}

\author{
Two-mode social networks: \\ conceptual aspects
}

Maria Inês TOMAÉL²

Regina Maria MARTELETO3

\section{Resumo}

As redes de dois modos compreendem as ligações entre dois conjuntos distintos de entidades; o termo "modo" refere-se a categorias específicas de entidades. Rede de um modo se caracteriza quando membros de uma rede têm ligações com outros membros da mesma categoria - como uma rede de pesquisadores -, e rede de dois modos se caracteriza quando seus membros possuem ligações com atores de outras categorias. A rede de dois modos é representada pela interação entre atores e entidades sociais como um único sistema social, como, por exemplo, as ligações entre pesquisadores e instituições (dois conjuntos de atores) em uma mesma rede. O objetivo deste artigo é conceituar e apresentar as redes de dois modos, que são empregadas nos estudos que utilizam a metodologia Análise de Redes Sociais. Por meio da análise da literatura, selecionaram-se artigos que enfatizam aspectos conceituais das redes sociais de dois modos, o que permitiu descrever e destacar exemplos e aplicações desse tipo de rede. No Brasil ainda são poucos os estudos publicados que mencionam ou empregam aplicações de Análise de Rede Sociais de dois modos. Com essa ênfase, este artigo contribui para disseminar aplicações de redes de dois modos.

Palavras-chave: Análise de redes sociais. Redes de afiliação. Redes sociais. Redes sociais de dois modos.

\begin{abstract}
Two-mode networks consist of connections between two distinct sets of entities. The term "mode" refers to specific categories ofentities. The term one-mode networks is used when members of a network are connected with other members in the same category-as a network of researchers - and the term two-mode network is used when members are connected with actors from other categories. The two-mode network is represented by the interaction among actors and social entities as a single social system, such as the connections between researchers and institutions (two sets of actors) within the same network. The purpose of this article is to conceptualize and present two-modenetworks, which are applied in studies that use Social Network Analysis. Through literaturereview, we selected articles thatemphasize conceptual aspects of two-mode social networks, which allowed us to describe and highlight examples and applications of this type of network. There are few published studies that mention or use two-mode Social Network Analysis in Brazil. Therefore, the goal of the article is to contribute to the promulgation of applications of two-mode networks.
\end{abstract}

Keywords: Social network analysis. Affiliation networks. Social networks. Two-mode social networks.

1 Resultados parciais do projeto desenvolvido no estágio de Pós-Doutorado de M.I.TOMAÉL, no Laboratório de Pesquisa em Comunicação e Saúde, Fundação Oswaldo Cruz. Apoio: Fundação Araucária, PR, Brasil.

2 Universidade Estadual de Londrina, Departamento de Ciência da Informação, Programa de Pós-Graduaçãoem Ciência da Informação. Rod. Celso Garcia Cid 445, km 380, Campus Universitário, 86051-990, Londrina, PR, Brasil. Correspondência para/Correspondence to: M.I. TOMAÉL. E-mail: <mitomael@uel.br>.

3 Instituto Brasileiro de Informação em Ciência eTecnologia - Universidade Federal do Rio de Janeiro, Programa de Pós-Graduação em Ciência da Informação. Rio de Janeiro, RJ, Brasil.

Recebido em 13/12/2012 e aceito para publicação em 7/3/2013 


\section{Introdução}

O estudo das relações sociais e da interação entre as pessoas é um dos interesses de pesquisa nas Ciências Sociais e, em especial, no escopo da Ciência da Informação. A análise de redes sociais insere-se nesse campo de estudo e é a metodologia que detém recursos de análise para conhecer e mapear as ligações entre indivíduos e entidades diversas.

A Análise de Redes Sociais (ARS) obteve progresso considerável no Brasil na última década. As aplicações mais comuns de ARS concentram-se em pesquisas de um modo, ou seja, os estudos presentes na literatura majoritariamente estudam redes de um modo. No Brasil, é incomum encontrar estudos que analisam redes de dois modos. Redes de um modo estudam um conjunto de atores similares, como pessoas, organizações, grupos sociais etc. Os estudos indicados exemplificam estudos de redes sociais de um modo: Análise de Redes Sociais aplicação nos estudos de transferência da informação (Marteleto, 2001); Redes de Conhecimento (Tomaél, 2005); Análise de Redes Sociais como metodologia de apoio para a discussão da interdisciplinaridade na Ciência da Informação (Matheus et al., 2006); Estudo da rede de coautoria e da interdisciplinaridade na produção científica com base nos métodos de análise de redes sociais (Silva et al., 2006); Redes Sociais de Informação - uma história e um estudo de caso (Ferreira, 2011).

Os estudos de redes de dados de um modo superam muito, em quantidade, os estudos de redes de dados de dois modos em todo o mundo. Na literatura científica, o número de trabalhos publicados sobre redes de dois modos é cerca de $80 \%$ menor do que de um modo.

Na Análise de Redes Sociais, os dados de dois modos referem-se às ligações registradas entre dois conjuntos de entidades. Neste contexto, Borgatti (2009) explica que o termo "modo" refere-se a uma classe de entidades - geralmente chamada de atores, nós ou vértices -, cujos membros têm ligações sociais com outros membros (no caso de um modo) ou com membros de outra classe (no caso de dois modos).

Com o objetivo de conceituar e apresentar as redes de dois modos, este artigo aborda o escopo dessas redes, que também são denominadas redes de afiliação ou bipartidas, e tem o intento de introduzir as possibili- dades de aplicação. Os argumentos incorporados a este artigo têm função didática e o intuito de aproximar os interessados em redes de dois modos aos conceitos elementares que Ihes são peculiares e, ainda, incluem exemplos de aplicações básicas para o estudo de redes que possuem duas ou mais categorias de atores.

O artigo foi elaborado com base na literatura científica, a partir da qual as publicações foram selecionadas intencionalmente e com base na expertise dos autores na temática e por sua inserção no campo de redes sociais. Inicialmente apresenta-se as abordagens de George Simmel para interação e círculos sociais que fundamentam a compreensão dos parâmetros específicos de redes de dois modos. Em seguida, resgata-se os processos de ARS para introduzir as redes de dois modos e suas peculiaridades para o estudo das ligações de atores e entidades sociais, conceituando e exemplificando aplicações iniciais.

\section{Interação e círculos sociais}

A interação e os círculos sociais são conceitos apresentados por George Simmel no início do século XX e, neste artigo, são relacionados aos conceitos que delineiam as redes de dois modos ou de afiliação.

A interação, intrínseca à sociedade, ocorre por meio de ações recíprocas entre indivíduos que constituem uma unidade, levando em consideração sempre determinados fins. A cooperação e a colaboração, na visão de Simmel (1983), estão associadas ao conceito de interação; isso porque apenas quando os indivíduos produzem ações que influenciam uns aos outros acontece a interação, em razão de impulsos ou propósitos. A importância da interação reside no fato de que é por meio dela que se forma a unidade; o agrupamento de indivíduos em torno de um empreendimento compreende, também, a formação de círculos sociais.

Os processos de interação e de sociação, para Simmel (1983), são considerados como básicos quando socialmente analisados. A interação é uma forma de sociação, conceito cunhado por simmel que trata do agrupamento de indivíduos em unidades por interesses comuns. É por meio da interação que pode-se observar os círculos sociais, interligações entre indivíduos de grupos distintos e dentro de um mesmo grupo. 
O primeiro círculo social do qual o indivíduo participa é o familiar, e é neste círculo que Simmel (2004) considera que as ligações são mais estreitas. Na família, o indivíduo fortalece os laços necessários à sua formação inicial, os quais abrangem as mais variadas individualidades. À medida que os indivíduos vão se desenvolvendo, criam ligações com membros de fora do seu círculo inicial de associação; com esses membros mantêm relacionamentos baseados na"[...] igualdade objectiva [sic] de predisposições, tendências, atividades etc. A associação através da convivência exterior vai sendo substituída cada vez mais pelas relações de conteúdo" (Simmel, 2004, p.574).

Com o desenvolvimento dessas ligações, diz Simmel, surgem outros círculos que possibilitam cruzamento de contatos inicialmente estabelecidos nos mais diversos ângulos. Desse modo, o indivíduo integra-se a múltiplos círculos sociais durante sua existência, inicialmente junto com a família, depois no meio acadêmico, profissional, de amigos e, por meio dos diferentes círculos sociais a que pertence, vai constituindo uma rede de contatos sociais.

Mediante a participação em distintos grupos, o indivíduo cria um sistema de coordenadas, de modo que, a cada novo grupo do qual passa a fazer parte, esse indivíduo amplia seu campo de ação - que lhe é peculiar. Quanto maior o número de grupos de que um indivíduo participa, tanto maior será a dificuldade de dois indivíduos apresentarem a mesma combinação de grupos, ou seja, menor será a probabilidade de os círculos se cruzarem em um mesmo ponto (Simmel, 2004).

A noção de círculo social foi delineada por Kadushin (1966) como um importante tipo de entidade social, sem uma lista de adesão formal, regras ou liderança. O círculo social é visto pelo autor, ressaltam Wasserman e Faust (1994), como uma entidade não observável, que deve ser inferida a partir de semelhanças comportamentais entre grupos de indivíduos. Uma importante percepção de Kadushin (1966) é que os círculos sociais proveem condições para o desenvolvimento de ligações interpessoais. Uma cultura avançada amplia os círculos sociais a que os indivíduos pertencem, mas, ao mesmo tempo, diz Kadushin (1966), o indivíduo é feito para confiar em seus próprios recursos e, com isso, pode ser privado de muitos suportes e vantagens associadas à sua afiliação a um grupo coeso.
Os aportes apresentados por Simmel (2004), referentes a círculos sociais, serviram de base para Kadushin (1966) fundamentar algumas proposições a respeito das funções dos círculos. Primeiro, diz Kadushin, círculos sociais podem destruir tipos tribais e até um grupo formado em um bairro específico, assim como organizações sociais. Historicamente, há um desenvolvimento a partir desses grupos de associações baseadas na livre escolha. Em segundo lugar, para os círculos sociais se desenvolverem, deve haver pontos focais em torno dos quais eles possam se formar. Os pontos focais mais comuns são: classes sociais; idade e sexo (tipos mais comuns de círculos); interesses intelectuais e humanísticos; autointeresse; família; religião e outros. Em terceiro lugar, nas sociedades modernas, os indivíduos tendem a pertencer a diferentes círculos, em parte porque alguns círculos são consequências de outros, em parte porque cada pessoa, por meio de uma pluralidade de meios, adquire algumas configurações únicas de círculos. Em quarto lugar, como nas proposições anteriores, Simmel vê círculos sociais como propriedade tanto de integração quanto de produção de conflito.

A formação de redes pela afiliação de indivíduos a grupos sociais é demarcada por Simmel em alguns dos seus trabalhos (Simmel, 1955, 1983); a partir deles, muitos pesquisadores das Ciências Sociais desenvolveram a teoria de Simmel e evidenciaram, em seus estudos, os processos de afiliação de indivíduos a coletividade, como, por exemplo, Kadushin (1966); Alba e Moore (1978); Wasserman e Faust (1994); Fontes e Stelzig (2004); Fontes (2007); Kadushin (2012).

A partir desses estudos, pode-se afirmar que Simmel é amplamente reconhecido como um dos primeiros sociólogos a discutir as implicações teóricas de afiliações de indivíduos com a coletividade - os círculos sociais. De forma simples, Simmel afirma que a afiliação a grupos múltiplos (por exemplo, família, organizações voluntárias, grupos ocupacionais) é fundamental na definição da identidade social de indivíduos. Para o autor, a interseção do indivíduo com grupos ocorre em virtude de sua afiliação a esses grupos (Wasserman; Faust, 1994).

A inserção de grupos sociais em contextos sócio-históricos é estruturada a partir da afiliação voluntária e por interesses próprios de participantes desses grupos, que, na contemporaneidade, são heterogêneos, o que 
possibilita a ligação de indivíduos em redes sociais com formações diversas. Essas circunstâncias trazem para o indivíduo "[...] uma possibilidade maior de se elegerem filiações institucionais e padrões de sociabilidade mais adequados aos gostos individuais" (Fontes, 2007, p.7).

\section{Análise de redes sociais}

Uma rede social é formada por um conjunto de atores (nós da rede) e suas ligações. A Análise de Redes Sociais (ARS) consiste no método que possibilita o estudo das redes sociais pelo mapeamento e descrição das ligações entre os atores.

As pesquisas sobre redes sociais, geralmente, estão voltadas para as propriedades estruturais que afetam o comportamento dos indivíduos, além dos efeitos dos atributos pessoais e dos padrões de relacionamentos mantidos e desenvolvidos na rede, que, para Wellman (1983), possibilitam oportunidades e constrangimentos por influenciar o acesso de pessoas e de instituições sobre alguns recursos, como informação, riqueza e poder. Algumas dessas análises estudam as formas complexas em que ocorrem as ligações, cujas relações múltiplas ligam membros de um mesmo sistema social. Outras análises se concentram em tipos de ligações específicas, com o intento de pesquisar seu padrão global em um sistema social.

Na concepção de Fontes e Stelzig (2004), subjacente ao conceito de redes sociais está a compreensão dos processos interativos e de um método de análise, mas a importância maior é imputada ao entendimento da estrutura social, que ocorre por meio dos estudos das interações e dos fenômenos que a permeiam. A inserção de um ator na rede e a posição que ocupa na estrutura analisada permitem o estudo das interconexões organizacionais, o que possibilita a análise de fenômenos complexos como as relações de poder, o fluxo de informação e a distribuição de recursos.

Borgatti e Everett (1997), como pesquisadores e estudiosos do método de ARS, acreditam que a Ciência Social tradicional estuda atributos de indivíduos (atributos monádicos) e a análise de redes estuda atributos de pares de indivíduos, que ocorre em relação a outro indivíduo (atributos diádicos). As relações sociais são um dos tipos de atributos diádicos. Os atributos diádicos, as relações sociais entre atores, são representados em matrizes quadradas de um modo. No entanto, destacam que os dados nas Ciências Sociais tradicionais são representados por matrizes de dois modos (retangulares).

Uma rede de dois modos ou de afiliação não possui relações diádicas. Faust (1997) explica que, na relação de afiliação, cada ator está ligado a uma entidade e cada entidade a um ator. As ligações entre atores de um mesmo conjunto são peculiares às redes de um modo.

Comum a todos estes pontos de vista está a ideia de que os atores são reunidos por sua participação conjunta em entidades sociais. Participação conjunta em entidades ${ }^{4}$ sociais, na visão de Wasserman e Faust (1994), não só fornece a oportunidade para os atores interagirem, mas também aumenta a probabilidade de desenvolverem ligações diretas. A sobreposição de membros do grupo permite o fluxo de informações entre os grupos e pode possibilitar a coordenação das ações dos grupos. O fato é que as entidades, na perspectiva da ARS, mantêm um conjunto de atores afiliados a elas e os atores, um conjunto de entidades com as quais estão afiliados: essa é uma característica distintiva das redes de afiliação, também denominadas redes de dois modos.

\section{Redes de dois modos}

Os dados de dois modos têm início com dois conjuntos, um de entidades sociais e outro de atores sociais, que são definidos pela participação dos atores sociais em pelo menos uma das entidades (Freeman; Duquenne, 1993). Redes de dados de dois modos são caracterizadas pelo estabelecimento de relações de proximidade entre os dois conjuntos distintos de objetos (Brusco, 2011).

Embora exista uma quantidade significativa de conceitos e ferramentas para analisar redes de um modo - considerados estudos clássicos por Latapy et al. (2008) -, há ainda uma falta de conceitos e ferramentas que atendam às necessidades de análise de redes de dois modos.

\footnotetext{
${ }^{4}$ Emprega-se o termo "entidade", também empregado (entities) por Borgatti e Everett (1997) e Borgatti (2009), para representar uma classe/categoria distinta de atores. Wasserman e Faust (1994) usam a palavra "eventos" com esse significado, mas, para evitar confusões, preferi-se não usá-la, visto que, na Língua Portuguesa, eventos usualmente designam encontros científicos, congressos, seminários etc.
} 
A possibilidade de análise de redes de dois modos ocorre quando pesquisadores identificam as relações entre classes de atores diferentes, como pessoas e organizações, ou pessoas e associações. Borgatti (2009) ilustra essa aplicação quando afirma que um pesquisador pode coletar dados sobre as comunidades virtuais das quais os funcionários de uma organização participam. Esses dados são frequentemente reconhecidos como de afiliações. As afiliações facultam as ligações entre entidades por meio de seus membros. Essas ligações são consideradas como condutoras de informação e é por meio delas que uma entidade recebe influência das outras.

Muitas das grandes redes do mundo real têm uma natureza de dois modos, de forma que seus nós podem ser separados em dois conjuntos e as ligações diretas são possíveis apenas entre os nós de cada um dos conjuntos. Por exemplo: a) leitores (conjunto 1) e os jornais que leem (conjunto 2) - as ligações possíveis podem ser encontradas entre leitores e diferentes jornais (por meio da leitura); b) pesquisadores (conjunto 1) e instituições (conjunto 2) - as ligações são constituídas por seu vínculo institucional e pelas parcerias entre pesquisadores e distintas instituições.

Segundo Borgatti (2009), a representação de uma rede em uma matriz de duas dimensões é considerada de dois modos se as linhas e as colunas apresentarem conjuntos diferentes de entidades - por exemplo, as linhas podem corresponder às pessoas, enquanto as colunas podem corresponder às organizações (matrizes retangulares). Em contraste, uma matriz é de um modo se as linhas e colunas referirem-se ao mesmo conjunto de entidades, como uma matriz de cidade (linha) por cidade (coluna) (matrizes quadradas).

As redes de dois modos, denominadas por Wasserman e Faust (1994) de redes de afiliação, consistem em informações sobre subconjuntos de atores que participam das mesmas atividades. Uma rede de afiliação contém informações sobre dois conjuntos de atores mais amplos do que pares. Assim, as redes de afiliação não podem ser analisadas cuidadosamente apenas observando pares ou díades de atores ou entidades: uma rede de afiliação representa a associação entre dois ou mais conjuntos de nós, de modo que cada conjunto é uma entidade social diferente. Por exemplo, uma rede de usuários de informação interligada compõe-se de um conjunto de nós que é formado por pessoas (os usuários) e outro conjunto de nós que é formado pelas Unidades de Informação (organizações). As ligações dos usuários com as Unidades de Informação ocorrem conforme o uso que fazem das unidades, delineando-se, desse modo, a rede de dois modos.

Em síntese, a rede de dois modos é representada pela interação entre atores e entidades sociais como um único sistema social, como, por exemplo, as ligações entre pesquisadores e instituições (dois conjuntos de atores) em uma mesma rede. No entendimento de Wang et al. (2009), as redes de dois modos referem-se a dois conjuntos distintos em termos gerais, como pessoas e associações, com uma ligação que significa que uma pessoa é membro de uma associação. Esse tipo de fenômeno social é caracterizado como a dualidade de pessoas e grupos, isto é, uma relação mutuamente constitutiva entre elementos de dois conjuntos distintos.

Tem havido um interesse crescente na análise de dados de redes de dois modos, também chamadas de redes bipartidas. Dados de dois modos são comuns em contextos de redes sociais. Borgatti (2009) indica alguns exemplos típicos de dois modos: pessoas relacionadas a eventos; pessoas relacionadas a grupos de pessoas; pessoas relacionadas a adjetivos pessoais; pessoas relacionadas a objetos.

O estudo de redes de afiliação não requer, segundo Wasserman e Faust (1994), a interação face a face entre atores de um local físico em particular, num ponto específico no tempo; é necessário que se tenha uma lista de atores afiliados a entidades específicas. Em um sentido mais geral, os autores afirmam que um ator é afiliado a uma entidade se, em termos substanciais, o ator pertence ao clube, participou de uma reunião, fez parte da diretoria ou administração de uma organização, é membro de uma comissão, foi para uma festa e assim por diante. Quando não existe ambiguidade, pode-se também dizer que o ator pertence, foi ou é membro de uma entidade, conforme a situação.

Dada a variedade de possibilidades e aplicações de redes de afiliação, Wasserman e Faust (1994) observaram na literatura três das principais razões para os estudos:

1) A afiliação individual a entidades provê ligações diretas entre atores e essas entidades. Tal tipo de estudo é 
o mais comum. A motivação para esse estudo está no fato de a afiliação de atores a entidades constituir uma ligação direta, quer entre os atores por meio de associações com entidades, quer entre as entidades por meio de membros comuns.

2) O contato entre indivíduos que participam de uma mesma entidade social provê condições de que vínculos entre pares de indivíduos tornem-se mais provável. Alguns pesquisadores têm tratado afiliações como a condição necessária que pode facilitar a formação de ligações entre pares de atores. A afiliação de indivíduos a grupos sociais provê a oportunidade para a influência interpessoal.

3) A importância da interação entre atores e entidades como um único sistema social, que pode ser representado por um modelo de relacionamento entre atores e entidades como um sistema completo (total). Assim, pode-se estudar a estrutura e as propriedades do sistema social composto pelas afiliações de atores com acontecimentos e entidades, como um todo.

Cada uma dessas três razões implica, para Wasserman e Faust (1994), uma abordagem distinta para análise de dados. A primeira motivação leva ao estudo ou a rede de um modo, com ligações entre pares de atores que convivem por suas afiliações a entidades, ou a rede de um modo de ligações entre pares de entidades que decorrem do que os atores possuem em comum. A segunda motivação implica que o pesquisador estude tanto as relações de dois modos, atores e entidades, quanto as relações de um modo entre pares de atores. A terceira motivação ocorre com a análise de ambos os modos simultaneamente, com foco das ligações entre eles.

\section{Matriz de redes de dois modos}

A mais simples apresentação de uma rede de dois modos é a matriz, que registra a afiliação de cada ator a cada entidade. Essa matriz, denominada por Wasserman e Faust (1994) de matriz de afiliação, registra para cada entidade os atores filiados a ela.

Cada linha da matriz indica a afiliação de um ator a entidades e cada coluna indica os membros relacionados à entidade. O Quadro 1 apresenta um exemplo hipotético, que registra a participação de seis atores em três entidades distintas; o número 1 indica a presença da ligação e o 0 indica a ausência da ligação - ou seja, nas linhas estão registradas as ligações/vínculos dos atores com as entidades.

Dois conjuntos/categorias de atores figuram no Quadro 1: o conjunto dos atores (A1, A2 ... A6) e o conjunto das entidades (E1, E2, E3). Observa-se na primeira linha que o ator 1 (A1) é membro da entidade 1 (E1) e da entidade 3 (E3) e que não é membro da entidade 2 (E2). Na coluna 2, observa-se que A1 e A4 são membros da E1 e A2, A3, A5 e A6 não são.

Outro exemplo pode ser descrito a partir de uma pesquisa em andamento sobre as ligações entre pesquisadores e universidades no exterior: a matriz de dois modos (retangular), Quadro 2, indica a presença ou ausência de ligações entre pesquisadores (P1 a P8) e universidades (E1 a E12). Os dados primários de interesse da rede são representados pela afiliação do pesquisador a diferentes universidades no exterior. Nessa Matriz observa-se que todos os pesquisadores mantêm algum tipo de contato com a universidade E8, e que o pesquisador P6 é o que tem o maior número de ligações com universidades no exterior.

Quadro 1. Matriz de afiliação atores/entidades sociais.

\begin{tabular}{lccc}
\hline Atores/Eventos & E1 & E2 & E3 \\
\hline A1 & 1 & 0 & 1 \\
A2 & 0 & 1 & 0 \\
A3 & 0 & 0 & 1 \\
A4 & 1 & 1 & 0 \\
A5 & 0 & 0 & 1 \\
A6 & 0 & 1 & 0 \\
\hline
\end{tabular}

Fonte: Elaborado pelas autoras (2012).

Quadro 2. Matriz de afiliação - pesquisadores e universidades.

\begin{tabular}{ccccccccccccc}
\hline & E1 & E2 & E3 & E4 & E5 & E6 & E7 & E8 & E9 & E10 & E11 & E12 \\
\hline P1 & 0 & 0 & 1 & 1 & 0 & 0 & 1 & 1 & 0 & 0 & 0 & 1 \\
P2 & 0 & 1 & 0 & 0 & 1 & 0 & 1 & 1 & 1 & 0 & 0 & 0 \\
P3 & 1 & 0 & 1 & 0 & 0 & 0 & 0 & 1 & 0 & 1 & 1 & 0 \\
P4 & 0 & 0 & 0 & 0 & 1 & 1 & 0 & 1 & 0 & 0 & 0 & 0 \\
P5 & 1 & 0 & 0 & 0 & 0 & 0 & 0 & 1 & 1 & 1 & 0 & 0 \\
P6 & 0 & 1 & 0 & 0 & 1 & 0 & 1 & 1 & 0 & 0 & 1 & 1 \\
P7 & 1 & 0 & 0 & 0 & 0 & 0 & 0 & 1 & 0 & 0 & 0 & 1 \\
P8 & 0 & 0 & 1 & 0 & 0 & 0 & 0 & 1 & 0 & 0 & 0 & 0 \\
\hline
\end{tabular}

Fonte: Elaborado pelas autoras (2012). 


\section{Gráfico bipartido}

Uma rede de dois modos pode também ser representada por um gráfico bipartido. Nesse gráfico, os nós são representados por dois conjuntos.

A diferença do gráfico bipartido com os gráficos clássicos reside no fato de que os nós estão em dois conjuntos distintos, e que as ligações são sempre entre um nó de um conjunto e um nó do outro conjunto. Nas palavras de Latapy et al. (2008), não pode haver qualquer ligação entre dois nós no mesmo conjunto. Muitas grandes redes do mundo real podem ser representadas naturalmente por um gráfico bipartido, que representa as redes de dois modos ou redes de afiliação, as quais descrevem grupos e seus membros.

A Figura 1 apresenta um exemplo de gráfico bipartido (centro), representando dois conjuntos de nós (de 1 a 4 e de A a F) em que os atores mantêm ligações com entidades. O gráfico da esquerda e o da direita representam as ligações entre os nós de um mesmo conjunto.

O gráfico bipartido do Quadro 1, representado pela Figura 2, demonstra as entidades em preto (E1, E2, E3) e os atores ( $A 1, A 2, A 3, A 4, A 5, A 6)$ em cinza, dois conjuntos que estão relacionados pelas ligações entre os atores e as entidades. Por exemplo, A1 está em contato indireto com A5 por meio de sua ligação com E3; os dois atores podem receber as mesmas informações, assim como o fluxo de informação que tem origem em A5 pode alcançar $A 1$.

A análise de redes de dois modos apresenta a dualidade de pessoas e entidades. No exemplo de diagrama representado na Figura 3 (que teve sua origem a partir da matriz indicada no Quadro 2), as pessoas são representadas por pesquisadores e as entidades por universidades no exterior. No gráfico bipartido (Figura 3), as universidades estão representadas em preto (quadrados) e os pesquisadores em cinza (círculos). A figura permite a identificação visual da estrutura das relações sociais na rede, na qual pode-se observar que as universidades estão integradas à rede pelas ligações que mantêm com os pesquisadores. Cabe ressaltar que as ligações em redes de dois modos só são possíveis entre categorias distintas; no caso da Figura 3, as ligações são mantidas entre pesquisadores e universidades.

Por meio desses exemplos pode-se visualizar os círculos sociais, que são construídos pela interação dos indivíduos com grupos sociais. Os círculos sociais possuem uma peculiar combinação de interação indireta baseada em interesses comuns que se juntam com um baixo grau de institucionalização, de acordo com Kadushin (1966).

As relações entre indivíduos que trabalham ou estudam em torno de uma mesma atividade podem ser desenvolvidas a partir dessa atividade, ou seja, a probabilidade de estarem ligados indiretamente, de criarem e expandirem seus círculos sociais é grande, assim como a possibilidade de formarem um grupo coeso, uma rede.

\section{Considerações Finais}

As redes de dois modos possibilitam ao pesquisador verificar a forma com que atores e entidades estão relacionados e, ainda, constatar que as entidades criam ligações entre os atores e, por sua vez, os atores criam ligações entre as entidades.

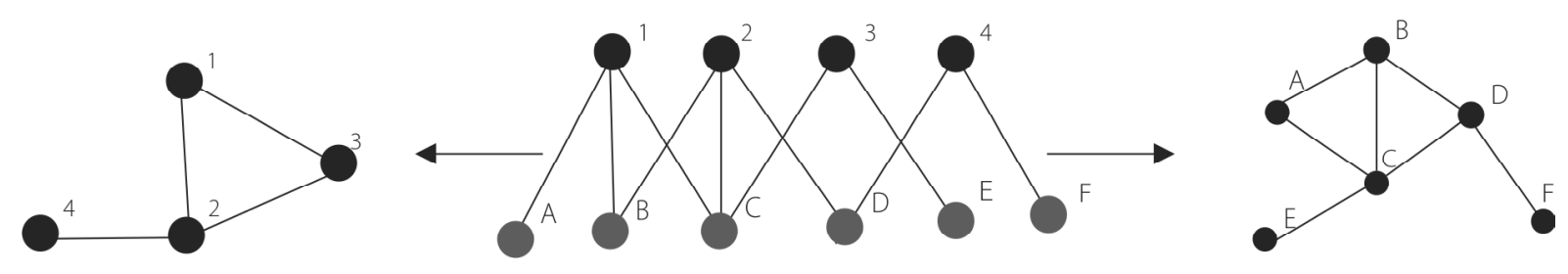

Figura 1. Exemplo de Gráfico Bipartido (centro).

Fonte: Latapy et al. (2008). 


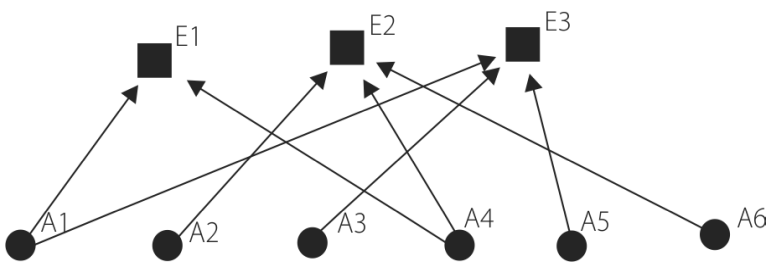

Figura 2. Gráfico bipartido da matriz apresentada no Quadro 1. Fonte: Elaborado pelas autoras (2012).

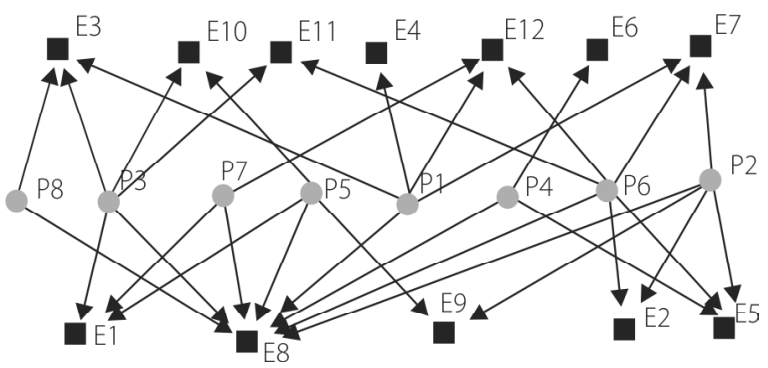

Figura 3. Gráfico bipartido na matriz apresentada no Quadro 2. Fonte: Elaborado pelas autoras (2012).

A decisão para a seleção de modos (um modo ou dois modos) para a análise de redes sociais é definida pela intenção do pesquisador. Os objetivos da pesquisa e o que se pretende alcançar ao final dela são determinantes para a definição do modo de análise em um estudo de redes.

Muitas são as aplicações possíveis para redes de dois modos ou de afiliações, como, por exemplo:

- Composição de conselhos acadêmicos - conjuntos: 1) membros, 2) conselhos;

- Membros de associações - conjuntos: 1) membros, 2) associações;

\section{Referências}

ALBA, R.D.; MOORE, G. Elite social circles. Sociological Methods \& Research, v.7, n.2, p.167-188, 1978.

BORGATTI, S.P. 2-Mode concepts in social network analysis. In: MEYERS, R.A. (Ed.). Encyclopedia of complexity and system science. Heidelberg: Springer, 2009. p.8279-8291.

BORGATTI, S.P.; EVERETT, M.G. Network analysis of 2-mode data. Social Networks, v.19, n.3, p.243-269, 1997.
- Pesquisadores e temas/campos de pesquisa - conjuntos: 1) pesquisadores, 2) campos/áreas do conhecimento;

Especificamente para o campo da Ciência da Informação, as aplicações que relacionam fluxos da informação e do conhecimento entre pessoas e entidades podem resultar em estudos profícuos para a área:

- Usuários de Unidades, sistemas ou Fontes de Informação - conjuntos: 1) usuários (pessoas), 2) Unidades, sistemas ou Fontes de Informações;

- Profissionais da informação atuantes em Unidades de Informação - conjuntos: 1) profissionais, 2) tipos de Unidades de Informação;

- Fluxo da informação entre mídias sociais - conjuntos: 1) participantes, 2) mídias sociais;

- Compartilhamento da informação pela participação de profissionais em eventos científicos - conjuntos: 1) profissionais, 2) eventos de que participaram.

A pluralidade de formas para a pesquisa e para a análise parece se ampliar indefinidamente; essa acepção provoca, no pesquisador, a necessidade de buscar pormenores e particularidades de procedimentos metodológicos que possam auxiliá-lo nos processos. Nessa perspectiva, este artigo é apenas um começo para a busca de novos conhecimentos.

O que está consolidado é a asserção de que um indivíduo participa de muitas redes sociais simultaneamente, nas quais seus círculos sociais se cruzam, e nessas redes representa papéis diferentes - na família, no trabalho, com amigos, na escola etc. As redes sociais são necessárias e imprescindíveis para a sociedade se manter coesa, à medida que aproximam elementos dispersos e transpõem ordens estabelecidas, como as geográficas e as hierárquicas.
BRUSCO, M. Analysis of two-mode network data using nonnegative matrix factorization. Social Networks, v.33, n.3, p.201-210, 2011

FAUST, K. Centrality in affiliation networks. Social Networks, V.19, n.2, p.157-191, 1997.

FERREIRA, G.C. Redes sociais de informação: uma história e um estudo de caso. Perspectivas em Ciência da Informação, v.16, n.3, p.208-231, 2011. 
FONTES, B.A.S.M. A construção das redes sociais de operadores de ONGs: os mecanismos de recrutamento a partir das relés sociais. REDES: Revista Hispana para el Análisis de Redes Sociales, v.12, p.1-27, 2007. Disponível em: <http://revistaredes.rediris.es/indicevol12.htm>. Acesso em: 18 nov. 2011.

FONTES, B.A.S.; STELZIG, S. Sobre trajetórias de sociabilidade: a ideia de relé social enquanto mecanismo criador de novas redes sociais. Política \& Sociedade, v.3, n.5, p.57-77, 2004. Disponível em: <https://periodicos.ufsc.br/index.php/ politica/article/view/1978>. Acesso em: 18 nov. 2011.

FREEMAN, L.C.; DUQUENNE, V. A note on regular colorings of two mode data. Social Networks, v.15, n.4, p.437-441, 1993.

KADUSHIN, C. The friends and supporters of psychotherapy: on social circles in urban life. American Sociological Review, v.31, n.6, p.786-802, 1966.

KADUSHIN, C. Understanding social networks: theories, concepts and findings. Oxford: Oxford University, 2012.

LATAPY, M.; MAGNIEN, C.; DEL VECCHIO, N. Basic notions for the analysis of large two-mode networks. Social Networks, v.30, n.1, p.31-48, 2008.

MARTELETO, R.M. Análise de redes sociais: aplicação nos estudos de transferência da informação. Ciência da Informação, v.30, n.1, p.71-81, 2001

MATHEUS, R. et al. Análise de redes sociais como metodologia de apoio para a discussão da interdisciplinaridade na ciência da informação. Ciência da Informação, v.35, n.1, p.72-93, 2006.

SILVA, A.B.O. et al. Estudo da rede de co-autoria e da interdisciplinaridade na produção científica com base nos métodos de análise de redes sociais. Encontros Bibli, n.esp., 2006. Disponível em: <http://www.periodicos.ufsc.br/index.php/ eb/article/view/1518-2924.2006v11nesp1p179>. Acesso em: 24 jan. 2012.

SIMMEL, G. Conflit and the web of group affiliations. New York: Free Press, 1955.

SIMMEL, G. Sociabilidade: um exemplo de sociologia pura ou formal. In: MORAES FILHO, E. (Org.). Georg Simmel: sociologia. São Paulo: Ática, 1983. p.165-181.

SIMMEL, G. O cruzamento de círculos sociais. In: CRUZ, M.B. (Org.). Teorias sociológicas: os fundadores e os clássicos. Lisboa: Fundação Calouste Gulbenkian, 2004. p.573-578.

TOMAÉL, M.I. Redes de conhecimento: o compartilhamento da informação e do conhecimento em consórcio de exportação do setor moveleiro. 2005. Tese (Doutorado em Ciência da Informação) - Escola de Ciência da Informação, Universidade Federal de Minas Gerais, Belo Horizonte, 2005.

WANG, P. et al. Exponential random graph $\left(p^{\prime \prime}\right)$ models for affiliation networks. Social Networks, v.31, n.1, p.12-25, 2009.

WASSERMAN, S.; FAUST, K. Social network analysis: methods and applications. Cambridge: Cambridge University, 1994.

WELLMAN, B. Network analysis: some basic principles. In: COLLINS, R. (Ed.). Sociological theory. San Francisco: Jossey-Bass, 1983. p.155-200. Available from: <http://www.bsos. umd.edu/ gvpt/CITE-IT/Documents/Wellman\%201983\%20Network\% 20Analysis.pdf>. Cited: Oct. 10, 2011. 

